\title{
Creation of a Balloon-Expandable Bifurcated Covered Stent to Treat a Complex Left Anastomotic Stricture in a Lung Transplant Recipient
}

\author{
Ryan Kern ${ }^{a} \quad$ Kenneth Sakata ${ }^{b} \quad$ Steve G. Peters ${ }^{a}$ David Midthun ${ }^{a} \quad$ John Mullon ${ }^{a}$ \\ Eric Edella
}

${ }^{a}$ Division of Pulmonary and Critical Care Medicine, Mayo Clinic, Rochester, MN, USA; ${ }^{b}$ Division of Pulmonary and Critical Care Medicine, Mayo Clinic, Scottsdale, AZ, USA

\section{Established Facts}

- In vivo creation of bifurcations in balloon-expandable metallic stents is a strategy used in interventional cardiology and vascular medicine to manage complex vascular stenosis.

\section{Novel Insights}

- In vivo creation of a bifurcated balloon-expandable covered stent may be an option to treat complex airway stenosis when other standard options have failed.

\section{Keywords}

Lung transplantation · Interventional pulmonology · Stent · Bronchoscopy

\begin{abstract}
Airway complications after lung transplant occur in approximately $10-15 \%$ of the recipients and often occur at the anastomosis, largely due to ischemia. To decrease anastomotic ischemia, surgeons minimize the length of the donor bronchus. However, a shortened donor bronchus creates technical challenges if a stent is required to treat an airway complication. We present a case of a lung transplant recipient with the combination of left main stem bronchial malacia and a triad of severe strictures at the left anastomosis, entrance to the left upper lobe, and left lower lobe. After failing several
\end{abstract}

\section{KARGER}

(c) 2018 S. Karger AG, Basel

E-Mail karger@karger.com

www.karger.com/res attempts using other modalities, success was achieved with in situ creation of a bifurcated fully covered balloon-expandable metallic stent. We describe a novel technique of punching a side branch hole through the wall of the stent to allow a left upper lobe stent to be placed through a stent directed into the left lower lobe in a $Y$ configuration with a good clinical outcome.

(c) 2018 S. Karger AG, Basel

\section{Introduction}

Airway complications remain a significant source of morbidity after lung transplant (LT) [1-3]. Many surgical techniques have been tried to minimize airway complications at the anastomosis. One currently preferred 


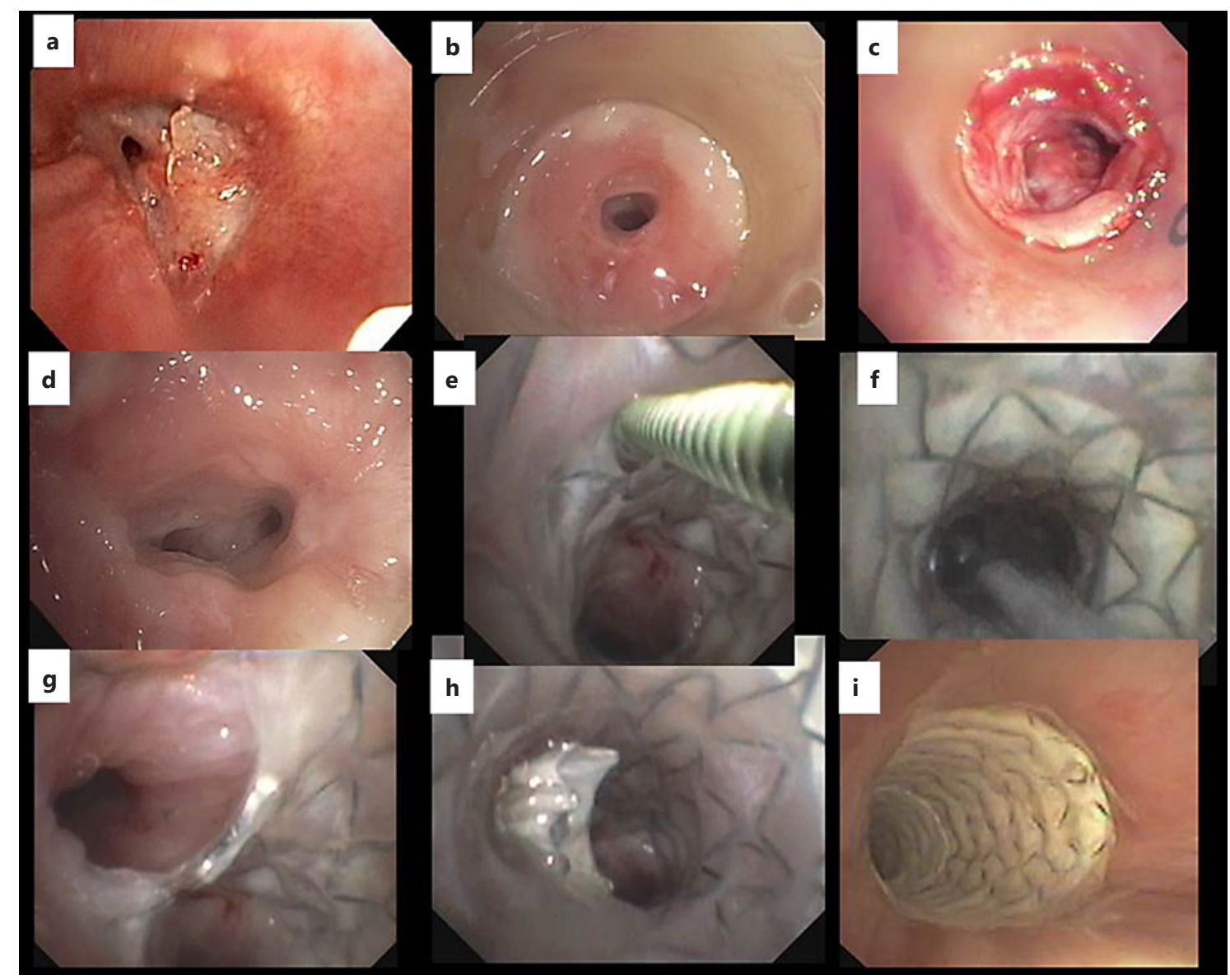

Fig. 1. Bronchoscopic images. a Initial anastomotic stricture. b Typical view at the distal end of the left main stem stent before dilations. c Typical view at the distal end of the left main stem stent after dilations. $\mathbf{d}$ Left upper lobe orifice on the left, left lower lobe orifice on the right after left main stem stent removal. e Hole in the
iCAST stent with guide wire passed into the left upper lobe. $\mathbf{f} 5 \times$ $20 \mathrm{~mm}$ balloon dilating hole to create access to the left upper lobe. g Hole in the iCAST stent revealing the left upper lobe. $\mathbf{h} 6 \times 16$ $\mathrm{mm}$ iCAST stent in the left upper lobe. i Proximal view of the left main stem, showing a seam between 2 iCAST stents. technique is to perform an end-to-end anastomosis minimizing the length of the donor bronchus as much as possible [4]. Though this technique may reduce airway complications, the shortened donor bronchus complicates stenting, particularly on the left side due to the bifurcation of the left upper lobe (LUL) and left lower lobe (LLL).

If a stent is needed on the left side, a straight stent may not be effective, and bifurcated silicone Y-stents with limbs going into the LUL and LLL have been used by our institution and others [5-7]. Upper lobe departure holes can be created in silicone stents, but for anatomical reasons, that strategy is usually more useful on the right than the left [8]. In cardiovascular medicine, bifurcations and side branches of blood vessels fre- quently pose a challenge for stent placement. Vascular interventionalists have developed techniques to "punch a hole" in the wall of a stent to allow blood flow or placement of another stent to create a bifurcated or "side branch" stent [9].

We present a case of an LT recipient with distal left main stem bronchial malacia with a triad of recurrent severe strictures of the anastomosis, LUL, and LLL orifices refractory to numerous interventions. We describe the successful treatment of this problem with the creation of a Y-configured bifurcated fully covered balloon-expandable covered metallic stent (BECMS) composed of 3 separate BECMS (iCAST Balloon Expandable Covered Stent; Atrium USA, Hudson, NH, USA). 


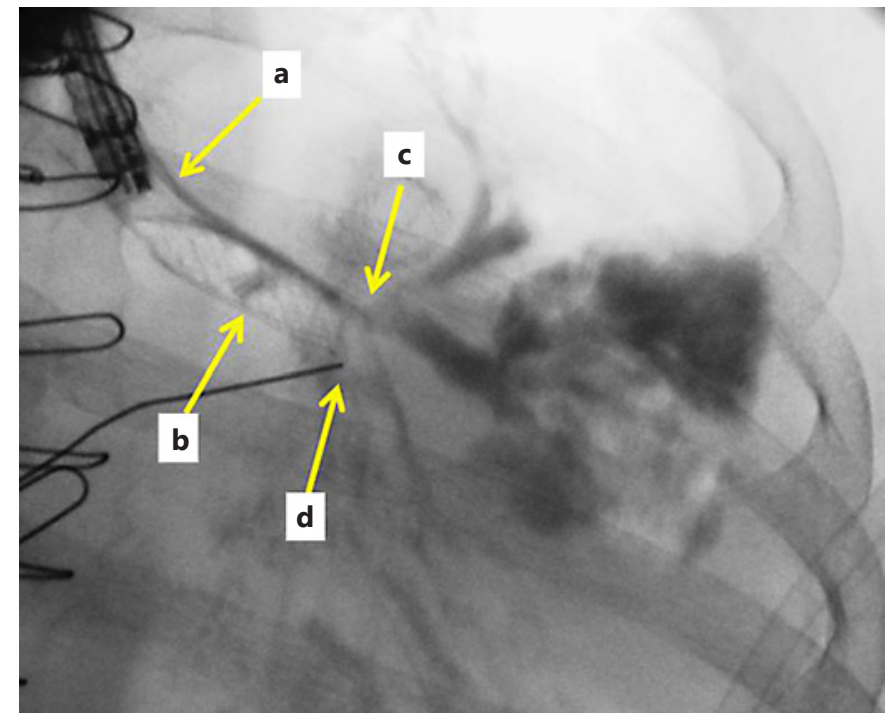

Fig. 2. Contrast bronchogram of the left upper lobe. a 4-Fr angled guide sheath initially placed into the left upper lobe through rigid bronchoscope so that contrast could be instilled into the left upper lobe to mark the left upper lobe orifice. b Deployed $8 \times 38 \mathrm{~mm}$ iCAST stent that had migrated back no longer going into the left lower lobe. c Orifice to the left upper lobe with upper division and lingular branches filled with contrast. d Marker used to indicate the proximal superior segment of the left lower lobe to avoid occluding with the distal end of the stent.

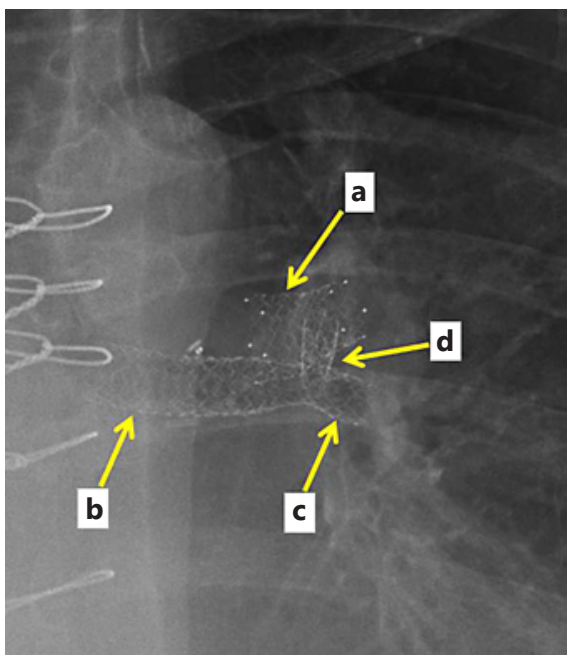

Fig. 3. Postprocedural chest X-ray. a Pulmonary artery stent. b Proximal left main stem bronchus stent, postdilated from 8 to 10 $\mathrm{mm}$. c Distal left main stem iCAST stent, $8 \mathrm{~mm}$ diameter. $\mathbf{d}$ Left upper lobe branch stent, $6 \mathrm{~mm}$ diameter.

\section{Case Report}

A 50-year-old female underwent bilateral LT for fibrotic lung disease secondary to usual interstitial pneumonia. Her post-LT course was complicated by pulmonary emboli, a stricture at the left pulmonary artery anastomosis treated with stenting of the pulmonary artery, and acute cellular rejection (grade A3). She developed a severe stricture at the left anastomosis by 90 days after LT (Fig. 1a). The stricture was treated with balloon dilation, rigid dilation, and finally placement of an LC2 Y stent 4 months after LT. The stent occluded with mucus multiple times and was removed 10 months after placement. Unfortunately, the anastomotic stricture recurred, and she developed new strictures to the LUL and LLL. Replacement of the silicone Y stent was not technically possible. The left main bronchus (LMB) malacia was treated with a straight stent, and the LUL and LLL strictures were treated with serial dilations. Because the patient was requiring bronchoscopy and dilation on a biweekly or monthly basis, we opted to try a novel technique of creating an in vivo custom stent with a side branch into the LUL.

\section{Procedure Description}

The patient was brought to the interventional pulmonary suite and was intubated with a rigid bronchoscope. The existing fully covered self-expanding metal stent was removed, the LUL and LLL were dilated with a small rigid bronchoscope (outer diameter 7.5 $\mathrm{mm}$ ), and we proceeded with a series of interventions.

We ultimately placed 3 BECMS, which come preloaded on a fixed diameter balloon but can be dilated further after deployment. The scaffold of the stent is made with stainless steel and is covered in 2 layers of polytetrafluoroethylene (PTFE) that completely cover the metal struts. The length of the stent slightly shortens as the stent is dilated. It is not self-expanding and maintains shape after dilation. We first placed an $8 \times 38 \mathrm{~mm}$ stent across the LMB stricture into the LLL. We dilated the proximal half to $10 \mathrm{~mm}$ using a $10 \times 20 \mathrm{~mm}$ vascular balloon (Charger vascular balloon; Boston Scientific, USA) which caused the stent to migrate proximally as the length shortened. A contrast bronchogram using a $50 \%$ mixture of Iohexol (Omnipauq 300; GE Healthcare, USA) and normal saline was performed so we would have a reference point to the LUL orifice (Fig. 2).

Because of the migration, we then telescoped a second $8 \times 38$ $\mathrm{mm}$ stent from the middle portion of the previously placed stent into the LLL, just overhanging the superior segment of the LLL. We then used an 8-mm-long 21-G peripheral biopsy needle (superDimension aspirating needle; Medtronic, USA) to make a small hole at the region of the LUL orifice. We used fluoroscopy to help us determine where to puncture. We advanced a steerable angled 0.035 " guidewire (Storq Steerable Guidewire; Cordis/Cardinal Health, USA) through the hole into the LUL under fluoroscopic guidance (Fig. 1e). A $5 \times 20 \mathrm{~mm}$ vascular balloon (Charger vascular balloon, Boston Scientific) was then inserted over the wire into the LUL and inflated to 12 atmospheres of pressure with a resulting hole (Fig. 1f, g). The steerable guidewire was essential in allowing us to make the angle. Finally, a $6 \times 16 \mathrm{~mm}$ stent was placed over the same wire into the LUL (Fig. 1h). A post-procedure chest X-ray demonstrates configuration (Fig. 3). The ends of the stent were flared by the use of forceps and gentle manipulation of a $5-\mathrm{mm}$ balloon. The resulting stents allowed patency of the entire LMB, LUL, and LLL. The superior segment of the LLL is partially occluded by the distal end of the stent. 


\section{Clinical Outcome}

The patients' $\mathrm{FEV}_{1}$ in $1 \mathrm{~s}$ improved from $1.51 \mathrm{~L}$ ( $55 \%$ predicted) to $1.95 \mathrm{~L}$ ( $70 \%$ predicted) immediately following stenting with dramatic improvement in exertional dyspnea and cough. At 5 months following the procedure, the $\mathrm{FEV}_{1}$ has remained stable at $2.01 \mathrm{~L}$ (73\% predicted). A follow-up bronchoscopy 5 months after the procedure showed minimal granulation tissue and patent stents. She has not had any complications in the 6 months after stent placement and has been maintained on 3\% inhaled saline 4 times per day. She does endorse intermittent coughing up cylindrical plugs of mucus.

\section{Discussion}

We present the first report of in vivo creation of a bifurcated BECMS to treat combined LMB malacia with a triad of strictures at the left anastomosis, LUL, and LLL in an LT recipient. Due to variations in anatomy between donors and recipients, there is not an ideal stent for all clinical scenarios. We have found that in some scenarios, the ability to post-dilate balloon-expandable stents may be advantageous for small airways and to address complex anatomical issues, such as in our case.

BECMS have reportedly been used to treat lobar stenosis [10]. Other bare metal balloon-expandable stents have been used to treat short lobar stenosis for nonanas- tomotic complications after LT [11]. To our knowledge, the creation of a hole in the wall of a BECMS with creation of a side branch has never been reported in the literature to treat a LT recipient. In situ customization of stents is established practice in cardiac and vascular interventions [9] but is a novel approach in the airway. It is unknown if this stent type and configuration will increase the risk of granulation tissue and mucus plugging. This will continue to be monitored. As the field of interventional pulmonology evolves, we expect that other techniques translated from other fields, such as our creation of a bifurcated stent, will become more common. The procedure that we performed was technically challenging, and we expect this to remain a salvage therapy for now.

\section{Statement of Ethics}

Institutional review board approval was not required for this study.

\section{Financial Disclosure and Conflicts of Interest}

Dr. Kern has a past consulting relationship with Olympus Corporation of the Americas and Auris Surgical Robotics.

\section{References}

1 Shofer SL, Wahidi MM, Davis WA, et al: Significance of and risk factors for the development of central airway stenosis after lung transplantation. Am J Transplant 2013;13: 383-389.

2 Machuzak M, Santacruz JF, Gildea T, Murthy SC: Airway complications after lung transplantation. Thorac Surg Clin 2015;25:55-75.

3 Sundset A, Lund MB, Hansen G, Bjortuft O, Kongerud J, Geiran OR: Airway complications after lung transplantation: long-term outcome of silicone stenting. Respiration 2012;83:245-252.

4 van Berkel V, Guthrie TJ, Puri V, et al: Impact of anastomotic techniques on airway complications after lung transplant. Ann Thorac Surg 2011;92:316-320; discussion 320-311.
5 Lee HJ, Puchalski J, Sterman DH, et al: Secondary carina Y-stent placement for postlung-transplant bronchial stenosis. J Bronchology Interv Pulmonol 2012;19:109-114.

6 Dutau H, Breen DP: Stenting the left secondary carina: "a fork in the road." Respiration 2015;90:441-442.

7 Oki M, Saka H: Silicone Y-stent placement on the secondary left carina. Respiration 2015; 90:493-498.

8 Breen DP, Dutau H: On-site customization of silicone stents: towards optimal palliation of complex airway conditions. Respiration 2009; 77:447-453.
9 Nairooz R, Saad M, Elgendy IY, et al: Longterm outcomes of provisional stenting compared with a two-stent strategy for bifurcation lesions: a meta-analysis of randomised trials. Heart 2017;103:1427-1434.

10 Majid A, Kheir F, Chung J, et al: Covered balloon-expanding stents in airway stenosis. J Bronchology Interv Pulmonol 2017;24:174177.

11 Fruchter O, Abed El Raouf B, Rosengarten D, Kramer MR: Long-term outcome of short metallic stents for lobar airway stenosis. J Bronchology Interv Pulmonol 2017;24:211215 